\title{
Inequality of the direct and consensual light reflexes in normal subjects
}

\author{
SHIRLEY A. SMITH, C. J. K. ELLIS, AND S. E. SMITH \\ From the Departments of Pharmacology and Medical Ophthalmology, St. Thomas's Hospital \\ and Medical School, London
}

SUMMARY Anisocoria in darkness and during reflex responses to unilateral light stimulation was studied in 150 normal subjects with television pupillometry. It was commonly found that the direct light reaction of the stimulated eye exceeded the consensual reaction of the other eye. This light-induced anisocoria, termed 'contraction anisocoria', had a mean value of $0.075 \mathrm{~mm}$ or $6.1 \%$ of light reflex amplitude. The measurement showed a high degree of repeatability in 20 subjects who were tested on two occasions a year apart. It occurred in the presence and absence of prior dark adaptation and increased proportionally with reflex amplitude as the intensity of the stimulating light was raised. It is concluded that, contrary to previous opinion, a small degree of contraction anisocoria is normal.

It is generally accepted that on unilateral light stimulation the direct and consensual light reflexes are of equal magnitude provided there is no lesion in the reflex pathways. However, Lowenstein (1954) described a syndrome, which he called 'alternating contraction anisocoria', in which the direct pupillary contraction of the illuminated eye in response to unilateral light stimulation exceeded the consensual contraction of the contralateral pupil. This lightinduced anisocoria was said to be 'alternating' because it varied according to which eye was stimulated. It was said to indicate an abnormality in the reflex pathways because it was found in a third of 'unselected clinical cases' (Lowenstein and Loewenfeld, 1969) but was rarely found in normal subjects. In this paper evidence is presented to show that contraction anisocoria is common in normal subjects, although the extent to which the direct reaction exceeds the consensual is small.

\section{Methods}

One hundred and fifty healthy subjects of both sexes, mean age 34.9 years (range 18 to 67 years) who had no history of eye disease volunteered for the study. Vertical pupillary diameters were measured with a Whittaker Corporation Series 1800

Address for reprints: Dr Shirley Smith, Department of Pharmacology, St. Thomas's Hospital Medical School, London SE1 7EH binocular infra-red television pupillometer (resolution $0.03 \mathrm{~mm}$ ) with the subject focusing on infinity. Light reflexes were elicited with light flashes of 100 or $500 \mathrm{~ms}$ duration, given every 8 seconds, with the light beam focused to a diameter of $1.8 \mathrm{~mm}$ in the plane of the pupil. The light source (Sylvania $2 \mathrm{~W}$ tungsten arc lamp) was displaced horizontally to the side of the visual axis, and the angle of incidence so formed was $7^{\circ} 31^{\prime}$ which resulted in a predominant illumination of the nasal retina. The intensity of the light was varied by insertion of Wratten neutral density filters. Responses of both pupils were recorded on paper and on tape for computer analysis. The amplitude of each reflex and the resting diameter at the foot of the reflex were measured directly from the paper or by computer.

\section{BILATERAL LIGHT STIMULATION}

The eyes of 72 subjects were stimulated alternately with $500 \mathrm{~ms}$ flashes of light of a standard intensity that gave submaximal pupillary reflex responses $(1.0$ to $1.5 \mathrm{~mm})$ for this stimulus duration. Three flashes were directed at each eye, and the values for reflex amplitude and resting diameter were averaged.

In order to measure the extent of the contraction anisocoria at different light intensities 18 subjects were dark-adapted by wearing red goggles for 30 minutes prior to testing. For each subject each eye was stimulated with at least 5 light flashes, of $100 \mathrm{~ms}$ duration, at 6 light intensities, starting with the lowest. The responses at each intensity were averaged 


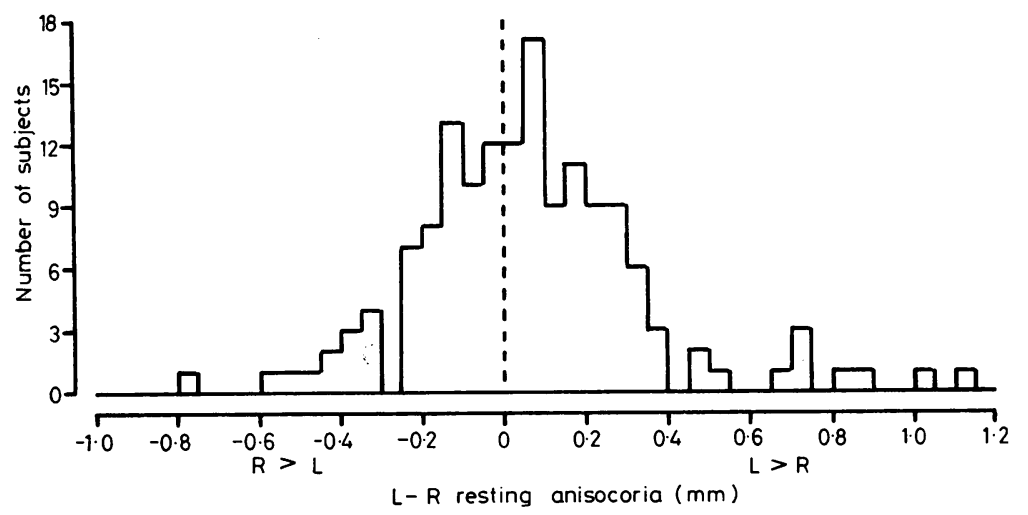

Fig. 1 Resting anisocoria in 150 normal subjects in the dark

Fig. 2 Left (L) and right $(\mathrm{R})$ reflex responses to 0.5 second light flashes directed at each eye $(l, r)$ alternately as indicated. The responses of the 2 eyes are separated on the time axis for convenience. Both subjects have contraction anisocoria (see text for explanation)

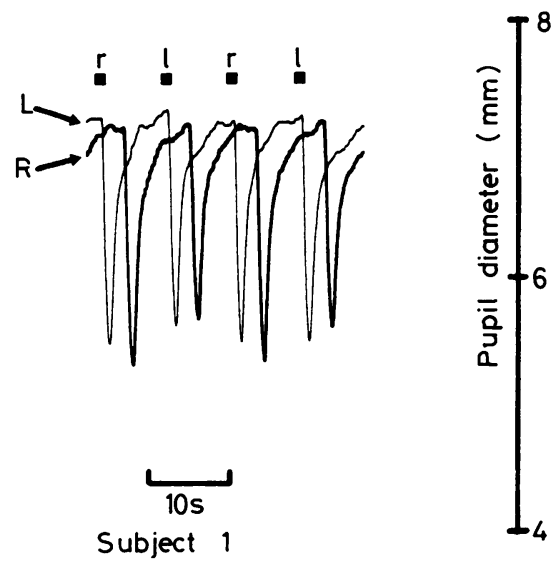

to obtain values for reflex amplitude and resting diameter.

UNILATERAL LIGHT STIMULATION

The left eye of 59 dark-adapted subjects was stimulated with light of an intensity that was adjusted for each individual's visual perception threshold (Fison et al., 1979). Six light reflexes were then elicited with $500 \mathrm{~ms}$ flashes of light of an intensity $10^{6}$ times greater than the perception threshold.

Twenty of these subjects were retested approximately 1 year later to assess the repeatability of the measured variables.

\section{Results}

RESTING ANISOCORIA

The normal range of resting anisocoria in darkness in all the subjects examined is shown in Fig. 1. The mean (left minus right diameter) for this population was $0.06 \mathrm{~mm}$, which is significantly different from zero $(\mathrm{P}<0.02)$. This indicates that there were more people with the left pupil larger than the right pupil than vice versa. However, as the repeatability of the measurement of resting anisocoria was not high (intrapair correlation coefficient $r=0.56$ ), this may have been a chance observation. In this population $95 \%$ had a resting anisocoria between 0 and $0.7 \mathrm{~mm}$ (irrespective of which pupil was larger), and those with larger values were clearly visible in dim light to the naked eye of the observer.

\section{CONTRACTION ANISOCORIA}

Three patterns of contraction anisocoria (when the direct reflex exceeded the consensual by more than $0.06 \mathrm{~mm}$, that is, twice the resolution of the pupillometer) were observed on bilateral light stimulation of 72 subjects:

(1) The bilateral form, found in $13(18.1 \%)$ subjects and illustrated in Subject 1 (Fig. 2). When the left eye of this subject was stimulated, the amplitude of the reflex in that eye exceeded the 
Fig. 3 Contraction anisocoria in 72 subjects on stimulation of (a) the right, and (b) the left eye

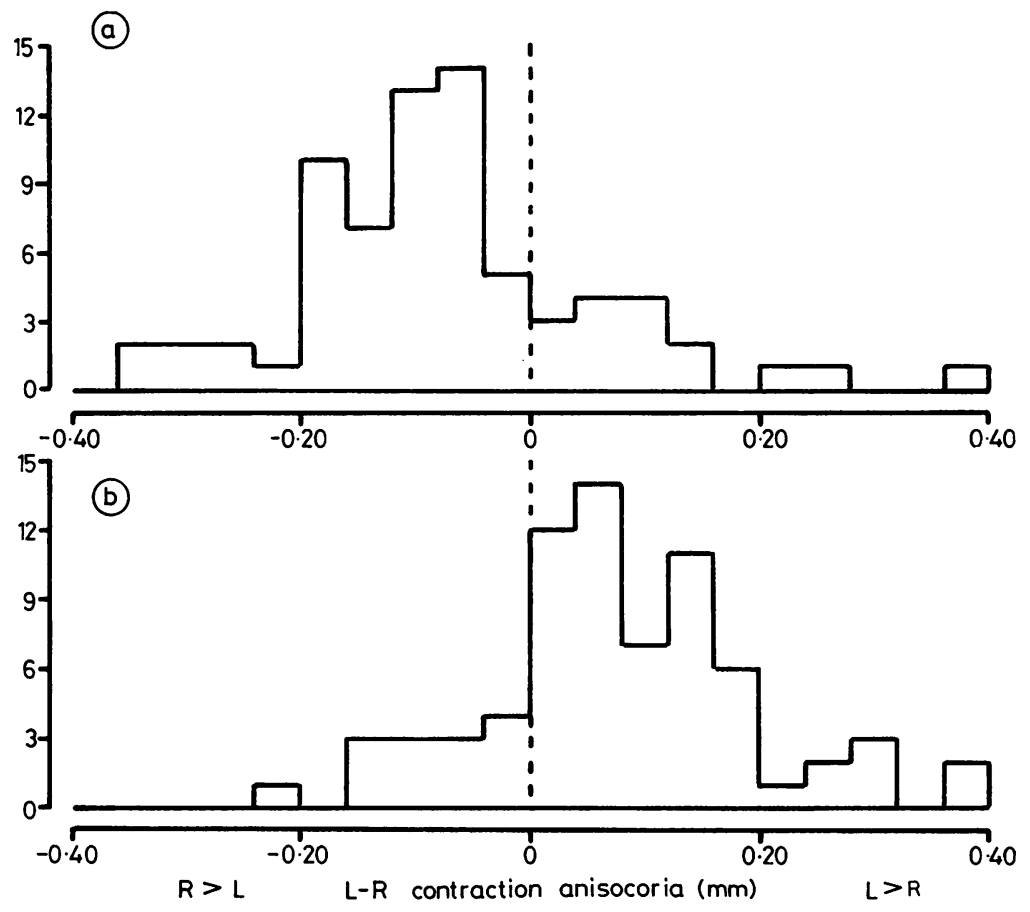

right by $0.13 \mathrm{~mm}$, but when the right was stimulated the right pupil contracted $0.11 \mathrm{~mm}$ more than the left.

(2) Unilateral contraction anisocoria, in which stimulation of only one side gave a greater direct response and stimulation of the other side resulted in equal reflex responses. This form occurred in 24 $(33.3 \%)$ subjects.

(3) A further 24 subjects had unilateral contraction anisocoria that differed from (2) in that stimulation of the second eye resulted in the consensual reflex exceeding the direct, such as in Subject 2 (Fig. 2). In this subject the right pupil always responded less than the left irrespective of the side of stimulation, possibly as a consequence of its smaller resting diameter. Thus on stimulation of the right eye the left consensual reflex exceeded the right direct one. However, this subject did have contraction anisocoria, because the amount by which the left reflex exceeded the right was increased from 0.27 to $0.42 \mathrm{~mm}$ (or from 16 to $26 \%$ of reflex amplitude) on switching the stimulating light from right to left.

There was evidence, therefore, of contraction anisocoria in $84.7 \%$ of this group. In no subject did the consensual response exceed the direct one on both left and right stimulation.

Fig. 3 shows histograms of the contraction anisocoria in this population. The mean direct minus consensual difference was 0.07 and $0.08 \mathrm{~mm}$ on left and right side stimulation respectively. These values are significantly greater than zero $(P<0.001)$ but do not differ significantly from each other. Therefore when left and right side stimulation are considered together, the average contraction anisocoria $(n=144)$ was $0.075 \pm 0.010 \mathrm{~mm}$ (mean \pm standard error). With each value being taken as a percentage of the average reflex amplitude of the left-right pair, it was found that in this population the direct response exceeded the consensual by 6.1 $\pm 0.8 \%$.

The mean contraction anisocoria resulting from unilateral light stimulation of 59 dark-adapted subjects at an intensity related to individual perception thresholds was $6 \cdot 8 \pm 1 \cdot 2 \%$ of reflex amplitude. This did not differ from the mean contraction anisocoria found in the 72 subjects described above who were not dark-adapted. Repeatability of the measurement of the contraction anisocoria obtained in 20 subjects tested on two occasions approximately 1 year apart was high: $r=0.82$.

The absolute degree of contraction anisocoria increased by a small but significant extent with increasing light intensity $(\mathrm{F}=3 \cdot 22, \mathrm{P}<0.05)$ (Fig. 4a). Reflex amplitude also increased with intensity $(\mathrm{F}=348.6, \mathrm{P}<0.001)$ (Fig. $4 b)$, so that contraction anisocoria, when expressed as a percentage of reflex 


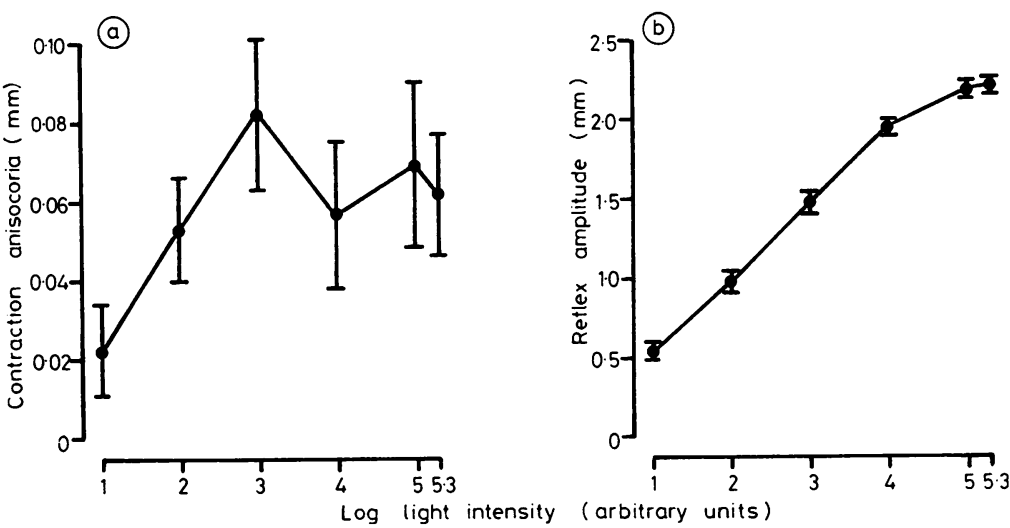

Fig. 4 The relationship between (a) contraction anisocoria and (b) reflex amplitude with increasing log light intensity. Means and standard errors $(\mathrm{n}=36)$ from stimulation of both eyes of 18 subjects are indicated amplitude, was not influenced by the brightness of the light.

\section{Discussion}

Lowenstein (1954) described the phenomenon of the direct light reflex exceeding the consensual as 'alternating contraction anisocoria'. The term 'alternating' is, however, misleading, because there are subjects in whom the contraction anisocoria does not reverse in direction when the light is switched from one to the other eye. Similarly the term 'consensual deficit' applied to the phenomenon by Thompson (1976) is not a comprehensive description, and we suggest therefore it be termed 'contraction anisocoria'.

Other authors have reported that contraction anisocoria is rare in normal subjects (Jones, 1949; Lowenstein and Loewenfeld, 1969) in contrast to the present study, where it occurred frequently. As the extent of the contraction anisocoria found in our normal population was small $(0.075 \mathrm{~mm}$ or $6.1 \%$ of reflex amplitude) it is possible that it was overlooked previously. Alternatively, it may be that it was even smaller with the brighter, longer light stimuli used by previous workers, although the results from this study (Fig. 4) do not support such a view. Another difference between this and previous studies concerns the position of the light source. In this study it was displaced slightly to the side of the visual axis, so that the nasal retina probably received more illumination than the temporal retina. Others have used larger, centrally placed lights giving more even retinal illumination. It would be interesting to study, therefore, whether discrete illumination of the temporal retina results in the same degree of contraction anisocoria reported here.

It is generally accepted that the supposed equality of the direct and consensual reflexes in man results from an approximately equal distribution of crossed and uncrossed fibres subserving pupillary function firstly in the optic chiasm and secondly in the midbrain. Further, it has been argued that the predominance of crossed fibres at both decussations in cats results in a markedly greater direct pupillary response in this species (Lowenstein et al., 1953). This suggests that the small contraction anisocoria reported here may result from an asymmetry in the decussations similar to, but smaller than that found in cats. There is some evidence that the chiasmal decussation in man may not be completely symmetrical. Kupfer et al. (1967) reported that the ratio of crossed to uncrossed fibres was 53:47 in a careful histological post-mortem examination of a case of unilateral enucleation. However, this study has yet to be confirmed, and further, the important anatomical evidence for this hypothesis, at present lacking, is the precise symmetry of the subsequent decussation in the midbrain.

The contraction anisocoria found in these normal subjects was usually too small to be seen without the aid of television pupillometry yet was remarkably consistent in individuals tested on separate occasions a year apart. In contrast, resting anisocoria was more variable with change in the size and even the direction of the anisocoria, a finding in agreement with a previous report (Loewenfeld, 1977). For clinical practice it is important to note, therefore, that the normal occurrence of contraction anisocoria may complicate the diagnosis of subtle pupillary defects by methods such as the swinging-light test (Thompson, 1976).

Two of the authors were in receipt of research fellowships from the Medical Research Council (SAS) and the Prevention of Blindness Research Fund (CJKE).

\section{References}

Fison, P. N., Garlick, D. J., and Smith, S. E. (1979). Assessment of unilateral afferent pupillary defects by pupil- 
lography. British Journal of Ophthalmology, 63, 195-199. Jones, I. S. (1949). Anisocoria: attempted induction by unilateral illumination. Archives of Ophthalmology, 42, 249-253.

Kupfer, C., Chumbley, L., and Downer, J. de C. (1967) Quantitative histology of optic nerve, optic tract and lateral geniculate nucleus of man. Journal of Anatomy, 101, 393-401.

Loewenfeld, I. E. (1977). 'Simple, central' anisocoria: a common condition, seldom recognized. Transcripts of the American Academy of Ophthalmology and Otolaryngology, 83, 832-839.
Lowenstein, O. (1954). Alternating contraction anisocoria. Archives of Neurology and Psychiatry, 72, 742-757.

Lowenstein, O., and Loewenfeld, I. E. (1969). The pupil. In The Eye, pp. 255-337. Edited by H. Davson. Academic Press: New York.

Lowenstein, O., Murphy, S. B., and Loewenfeld, I. E. (1953). Functional evaluation of the pupillary light reflex pathways: experimental pupillographic studies in cats. Archives of Ophthalmology, 49, 656-670.

Thompson, H. S. (1976). Pupillary signs in the diagnosis of optic nerve disease. Transactions of the Ophthalmological Society of the United Kingdom, 96, 377-381. 